\title{
Neutrophil-to-lymphocyte ratio in inflammatory bowel disease - As a new predictor of disease severity
}

\author{
Acarturk $\mathrm{G}^{1}$, Acay $\mathrm{A}^{2}$, Demir $\mathrm{K}^{1}$, Ulu $\mathrm{MS}^{3}$, Ahsen $\mathrm{A}^{2}$, Yuksel $\mathrm{S}^{3}$ \\ Afyon Kocatepe University, Faculty of Medicine, Department of Internal Medicine, Afyonkarahisar, Turkey. \\ drakifacay@gmail.com
}

\begin{abstract}
Background: The aims of this study were to investigate the utility of neutrophil-to-lymphocyte ratio (NLR) as a simple and readily available predictor for clinical disease activity in inflammatory bowel disease (IBD). Methods: Sixty-six patients (22 CD, $44 \mathrm{UC}$ ) and 41 healthy controls were enrolled in the study. Erythrocyte sedimentation rate (ESR), C-reactive protein (CRP) levels and complete blood count (CBC) were measured. The neutrophil and lymphocyte counts were recorded and NLR was calculated. The patients with active or inactive UC and CD were classified according to the severity of the disease.

Result: The serum NLR values of active CD patients were significantly higher than those of inactive ones and controls ( $p=0.000, p=0.000$, respectively). NLR values of active UC patients were significantly higher than those of inactive ones and controls $(p=0.000, p=0.000$, respectively. The optimum NLR cut-off point for active $C D$ and $U C$ was 3.2 and 3.1.

Conclusion: This study demonstrates that NLR in subjects with IBD is strongly associated with active disease and correlated with clinical and laboratory indices (Tab. 5, Fig. 2, Ref. 31). Text in PDF www.elis.sk. Key words: neutrophil-to-lymphocyte ratio, disease severity, new predictor, inflammatory bowel disease.
\end{abstract}

\section{Introduction}

Inflammatory bowel disease (IBD) has been a global healthcare problem with a sustained increasing incidence (1). It includes two major forms, Crohn's disease (CD) and ulcerative colitis (UC), which are distinct chronic bowel-relapsing inflammatory disorders. In a non-continuous type, $\mathrm{CD}$ can cause transmural inflammation and affect any part of the gastrointestinal tract (most commonly, the terminal ileum or perianal region). Unlike UC, CD is commonly associated with complications such as abscesses, fistulas and strictures. In contrast, UC is typified by mucosal inflammation and limited to the colon (2). After the diagnosis, it is crucial to monitor the disease via clinical, endoscopic, and laboratory indices of inflammatory activity in CD. Among the indices of clinical activity, the CDAI (Crohn's disease activity index) and Harey-Bradshow index (HDI) are the most widely used (3-8). CDAI is considered the gold standard for assessing the activity of the disease because this validated index has been widely used for more than 25 years in clinical protocols and in studies seeking drug approval (9). It is important to determine disease activity of UC early as this will significantly reduce the surgery rate, and therefore reduce mortality

${ }^{1}$ Afyon Kocatepe University, Faculty of Medicine, Department of Internal Medicine, Afyonkarahisar, Turkey, ${ }^{2}$ Afyon Kocatepe University, Faculty of Medicine, Department of Internal Medicine, Afyonkarahisar, Turkey, and ${ }^{3}$ Afyon Kocatepe University, Faculty of Medicine, Department of Nephrology, Afyonkarahisar, Turkey

Address for correspondence: A. Acay, Dr, Department of Internal Medicine, Afyon Kocatepe University Faculty of Medicine, Afyon Kocatepe Universitesi, Tip Fakultesi, Ic Hastaliklari AD, 03200, Afyonkarahisar, Turkey. Phone: +0090.505 .5066064 , Fax: +0090.272 .2463322$ in patients with serious UC (10). Noninvasive markers, such as Creactive protein (CRP), erythrocyte sedimentation rate (ESR), and white blood cells (WBCs) are widely acknowledged as important for both initial diagnosis and precise monitoring of disease activity in UC (11). Nevertheless, no optimal test has been developed so far. Therefore, the adjunctive use of additional serum markers may add a significant advantage for predicting disease severity and achieving diagnostic precision. Blood neutrophil-to-lymphocyte (NLR) ratio is a simple marker of subclinical inflammation that can be easily obtained from the differential WBC count. In recent years, neutrophilia and relative lymphocytopenia were shown to be independent predictors of mortality in patients with acute heart failure (12-14). In addition, recent studies introduce NLR as a potential marker to determine inflammation in both cardiac and non-cardiac disorders (15-18). The aims of this study were to investigate the utility of NLR as a simple and readily available predictor for clinical disease activity in IBD.

\section{Materials and methods}

\section{Patients and methods}

This retrospective study evaluated the diagnostic value of NLR for disease activity in 63 IBD (21 CD, $42 \mathrm{UC}$ ) patients managed at the Internal Medicine and Gastroenterology Departments of Afyon Kocatepe University Faculty of Medicine Hospital between September 2008 and August 2013. The control group consisted of 41 healthy subjects matched as to their age and gender (male/ female - 14/27).

Although 137 IBD patients were retrospectively reviewed, only 63 patients met study criteria. Patients were excluded from 
the study if they had hypertension, any acute inflammation, infection, acute or chronic renal failure, chronic liver or heart diseases, coronary artery disease, connective tissue diseases or were drug users with changing number of leukocytes.

The diagnosis of IBD was based on standard clinical, radiological, endoscopic and histological criteria. The data were extracted from the hospital database as follows - age, sex, localization of the disease, disease duration, drug intake and other medical history. Complete blood count (CBC), ESR, and CRP were also recorded for each IBD patient. All CBC analyses were performed in the hematology laboratory of our hospital. CBC analysis was performed with the same analyzer within two hours from collection of blood samples with the use of a Beckman Coulter (High Wycombe, UK) Gen-S automated analyzer. NLR was calculated from the differential count by dividing the absolute neutrophil count by the absolute lymphocyte count. Conditions possibly affecting NLR, such as prior treatment with corticosteroids, hematological or neoplastic disorders, diabetes mellitus, acute and chronic renal failure, rheumatic diseases and clinical evidence of active infection were defined as exclusion criteria for entry into the study. The study was conducted in accordance with the guidelines of the Helsinki declaration.

\section{Disease activity}

The disease activity in UC patients was assessed by Truelove and Witts criteria. These criteria have been validated for over 50 years and allow a simple and rapid stratification of patients with UC. Using this classification, UC patients were classified as mild, moderate or severe based on the number of bloody stools per day, heart rate, hemoglobin, ESR, and body temperature. Patients categorized as having moderate or severe disease were accepted as having active UC, whereas the patients in the mild group were considered to be in the remission period. The activity of Crohn's disease was evaluated by the physician with Harvey-Bradshaw index (1980) which is strongly correlated with Crohn's disease activity index (CDAI) and is easier to use. It evaluates five clinical parameters including well-being, abdominal pain, number of liquid stools each day, abdominal mass, and complications. A score inferior to 4 indicates an inactive disease while that above 4 indicates that the disease is active. The patients included in the present study within the activation period were reassessed two months after clinical remission. NLR values of IBD patients in whom clinical remission was achieved were noted.

\section{Statistical analysis}

The Statistical Package for Social Sciences (SPSS) 18.0 for Windows was used to analyze the data. Continuous variables were tested for normality by the Kolmogorov-Smirnov test. Values were presented as mean \pm standard deviation or in case of non-normally distributed data, as median and range. Comparisons of percentages between different groups of patients were carried out using the chi $^{2}$ test. All normally distributed data were analyzed using unpaired or paired student $t$ test. Data found to be non-normally distributed were analyzed using Mann-Whitney U test for independent subgroups, and Wilcoxon test for dependent subgroups.
Receiver operating characteristic (ROC) curve analysis was used to identify optimal cut-off values of NLR, WBC, CRP and ESR with maximum sensitivity and specificity for differentiation of activation of IBD from remission. Spearman's correlation analysis was done between NLR and other inflammation markers. A p value of less than 0.05 was deemed statistically significant.

\section{Results}

The mean age of CD and control groups was $34.40 \pm 12.02$ and $39.33 \pm 7.77$ years, respectively $(\mathrm{p}=0.847)$. The mean age of the UC and control groups was $39.40 \pm 11.15$ and $39.33 \pm 7.77$ years, respectively $(\mathrm{p}=0.173)$. There were no statistically significant differences in age between study participants. The demographic and laboratory features of $\mathrm{CD}, \mathrm{UC}$ and control group are

Tab. 1. Demographic and laboratory features of $C D$ (active, inactive) patients and controls.

\begin{tabular}{|c|c|c|c|c|}
\hline & & $\begin{array}{c}\mathrm{CD} \\
(\mathrm{n}=21)\end{array}$ & $\begin{array}{c}\text { Control } \\
(\mathrm{n}=41)\end{array}$ & $\mathrm{p}$ \\
\hline & Age (year) & $34.40 \pm 12.02$ & $39.33 \pm 7.77$ & 0.847 \\
\hline & Gender (F/M) & $9 / 12$ & $27 / 14$ & 0.085 \\
\hline \multirow{6}{*}{$\begin{array}{l}\text { Active } \\
\text { period }\end{array}$} & CRP (mg/dl) & $3.21 \pm 3.98$ & $0.46 \pm 0.34$ & 0.022 \\
\hline & ESR $(\mathrm{mm} / \mathrm{h})$ & $43.52 \pm 21.71$ & $10.29 \pm 5.11$ & $<0.001$ \\
\hline & $\mathrm{WBC}\left(\mathrm{mm}^{3} \mathrm{x} 10^{3}\right)$ & $12.67 \pm 3.22$ & $6.85 \pm 1.44$ & $<0.001$ \\
\hline & Neutrophil & $10.03 \pm 3.22$ & $3.77 \pm 1.06$ & $<0.001$ \\
\hline & Lymphocytes & $2.61 \pm 0.48$ & $2.34 \pm 0.53$ & 0.005 \\
\hline & NLR & $5.25 \pm 1.85$ & $1.68 \pm 0.59$ & $<0.001$ \\
\hline \multirow{6}{*}{$\begin{array}{l}\text { Inactive } \\
\text { period }\end{array}$} & CRP (mg/dl) & $0.79 \pm 0.63$ & $0.46 \pm 0.34$ & NS \\
\hline & ESR $(\mathrm{mm} / \mathrm{h})$ & $12.05 \pm 8.52$ & $10.29 \pm 5.11$ & NS \\
\hline & $\mathrm{WBC}\left(\mathrm{mm}^{3} \times 10^{3}\right)$ & $7.7 \pm 1.1$ & $6.85 \pm 1.44$ & 0.005 \\
\hline & Neutrophil & $4.58 \pm 0.66$ & $3.77 \pm 1.06$ & 0.001 \\
\hline & Lymphocytes & $2.61 \pm 0.48$ & $2.34 \pm 0.53$ & 0.034 \\
\hline & $\overline{\text { NLR }}$ & $1.78 \pm 0.32$ & $1.68 \pm 0.59$ & NS \\
\hline
\end{tabular}

Mann-Whitney U Test was used. CD - Crohn disease, CRP - C-reactive protein, ESR - erythrocyte sedimentation rate, WBC - white blood cells, NLR - neutrophil lymphocyte ratio, NS - non-significant.

Tab. 2 Demographic and laboratory features UC (active, inactive) patients and controls.

\begin{tabular}{|c|c|c|c|c|}
\hline & & $\begin{array}{c}\mathrm{UC} \\
(\mathrm{n}=42)\end{array}$ & $\begin{array}{c}\text { Control } \\
(\mathrm{n}=41)\end{array}$ & $\mathrm{p}$ \\
\hline & Age (year) & $39.40 \pm 11.15$ & $39.33 \pm 7.77$ & 0.173 \\
\hline & Gender (F/M) & $14 / 28$ & $27 / 14$ & 0.003 \\
\hline \multirow{6}{*}{$\begin{array}{l}\text { Active } \\
\text { period }\end{array}$} & $\mathrm{CRP}(\mathrm{mg} / \mathrm{dl})$ & $3.99 \pm 5.26$ & $0.46 \pm 0.34$ & $<0.001$ \\
\hline & $\overline{\operatorname{ESR}}(\mathrm{mm} / \mathrm{h})$ & $37.24 \pm 21.50$ & $10.29 \pm 5.11$ & $<0.001$ \\
\hline & $\overline{\mathrm{WBC}}\left(\mathrm{mm}^{3} \times 10^{3}\right)$ & $11.14 \pm 2.15$ & $6.85 \pm 1.44$ & $<0.001$ \\
\hline & Neutrophil & $8.57 \pm 1.89$ & $3.77 \pm 1.06$ & $<0.001$ \\
\hline & Lymphocytes & $2.22 \pm 0.67$ & $2.34 \pm 0.53$ & NS \\
\hline & NLR & $4.12 \pm 1.41$ & $1.68 \pm 0.59$ & $<0.001$ \\
\hline \multirow{6}{*}{$\begin{array}{l}\text { Inactive } \\
\text { period }\end{array}$} & $\mathrm{CRP}(\mathrm{mg} / \mathrm{dl})$ & $0.57 \pm 0.61$ & $0.46 \pm 0.34$ & NS \\
\hline & $\overline{\operatorname{ESR}(\mathrm{mm} / \mathrm{h})}$ & $11.21 \pm 6.69$ & $10.29 \pm 5.11$ & NS \\
\hline & $\mathrm{WBC}\left(\mathrm{mm}^{3} \times 10^{3}\right)$ & $7.34 \pm 1.41$ & $6.85 \pm 1.44$ & NS \\
\hline & Neutrophil & $4.35 \pm 0.94$ & $3.77 \pm 1.06$ & 0.008 \\
\hline & Lymphocytes & $2.91 \pm 0.64$ & $2.34 \pm 0.53$ & $<0.001$ \\
\hline & NLR & $1.55 \pm 0.45$ & $1.68 \pm 0.59$ & NS \\
\hline
\end{tabular}

Mann-Whitney U Test was used. UC - ulcerative colitis, CRP - C-reactive protein, ESR - erythrocyte sedimentation rate, WBC - white blood cells, NLR - neutrophil lymphocyte ratio, NS - non-significant. 
Tab. 3. Laboratory features of IBD (active, inactive) patients.

\begin{tabular}{|c|c|c|c|c|}
\hline & & $\begin{array}{l}\text { Active } \\
\text { Period }\end{array}$ & $\begin{array}{l}\text { Inactive } \\
\text { Period }\end{array}$ & $\mathrm{p}$ \\
\hline \multirow{6}{*}{$\begin{array}{l}\mathrm{CD} \\
(\mathrm{n}=21)\end{array}$} & CRP (mg/dl) & $3.21 \pm 3.98$ & $0.79 \pm 0.63$ & 0.03 \\
\hline & $\overline{\operatorname{ESR}(\mathrm{mm} / \mathrm{h})}$ & $43.52 \pm 21.71$ & $12.05 \pm 8.52$ & $<0.001$ \\
\hline & $\mathrm{WBC}\left(\mathrm{mm}^{3} \times 10^{3}\right)$ & $12.67 \pm 3.22$ & $7.7 \pm 1.1$ & $<0.001$ \\
\hline & Neutrophil & $10.03 \pm 3.22$ & $4.58 \pm 0.66$ & $<0.001$ \\
\hline & Lymphocytes & $2.61 \pm 0.48$ & $2.61 \pm 0.48$ & $<0.001$ \\
\hline & NLR & $5.25 \pm 1.85$ & $1.78 \pm 0.32$ & $<0.001$ \\
\hline \multirow{6}{*}{$\begin{array}{l}\mathrm{UC} \\
(\mathrm{n}=42)\end{array}$} & CRP (mg/dl) & $3.99 \pm 5.26$ & $0.57 \pm 0.61$ & $<0.001$ \\
\hline & ESR $(\mathrm{mm} / \mathrm{h})$ & $37.24 \pm 21.50$ & $11.21 \pm 6.69$ & $<0.001$ \\
\hline & $\overline{\mathrm{WBC}}\left(\mathrm{mm}^{3} \times 10^{3}\right)$ & $11.14 \pm 2.15$ & $7.34 \pm 1.41$ & $<0.001$ \\
\hline & Neutrophil & $8.57 \pm 1.89$ & $4.35 \pm 0.94$ & $<0.001$ \\
\hline & Lymphocytes & $2.22 \pm 0.67$ & $2.91 \pm 0.64$ & $<0.001$ \\
\hline & NLR & $4.12 \pm 1.41$ & $1.55 \pm 0.45$ & $<0.001$ \\
\hline
\end{tabular}

Mann-Whitney U Test was used. IBD - Inflammatory bowel disease, CD - Crohn disease, UC - ulcerative colitis, CRP - C-reactive protein, ESR - erythrocyte sedimentation rate, WBC - white blood cells, NLR - neutrophil-lymphocyte ratio, NS - non-significant.

Tab. 4. Spearman correlation coefficients between NLR and other inflammation markers in patients with $\mathrm{CD}$ and UC.

\begin{tabular}{|c|c|c|c|c|c|c|c|c|}
\hline & & & & CRP & ESR & WBC & Neutrophil & Lymphocytes \\
\hline \multirow{4}{*}{$\mathrm{CD}$} & \multirow{2}{*}{ Active } & \multirow{2}{*}{ NLR } & $r_{s}$ & -0.061 & 0.242 & 0.242 & 0.882 & -0.262 \\
\hline & & & $\mathrm{P}$ & 0.793 & 0.291 & 0.000 & 0.000 & 0.252 \\
\hline & \multirow{2}{*}{ Inactive } & \multirow{2}{*}{ NLR } & $r_{s}$ & -0.022 & -0.042 & -0.135 & 0.409 & -0.569 \\
\hline & & & $\mathrm{P}$ & 0.924 & 0.856 & 0.561 & 0.066 & 0.007 \\
\hline \multirow{4}{*}{ UC } & \multirow{2}{*}{ Active } & \multirow{2}{*}{ NLR } & $\mathrm{r}_{\mathrm{s}}$ & 0.116 & 0.051 & 0.260 & 0.412 & -0.615 \\
\hline & & & $\mathrm{P}$ & 0.463 & 0.750 & 0.096 & 0.007 & $<0.001$ \\
\hline & \multirow{2}{*}{\multicolumn{2}{|c|}{ Inactive NLR - }} & & -0.198 & 0.200 & 0.266 & 0.515 & -0.451 \\
\hline & & & $\mathrm{P}$ & 0.208 & 0.203 & 0.089 & 0.000 & 0.003 \\
\hline
\end{tabular}

NLR - neutrophillymphocyte ratio, CD - Crohn disease, UC - ulcerative colitis, $\mathrm{CRP}$-C-reactive protein, ESR - erythrocyte sedimentation rate, $\mathrm{WBC}$ - white blood cells, NS - non-significant.

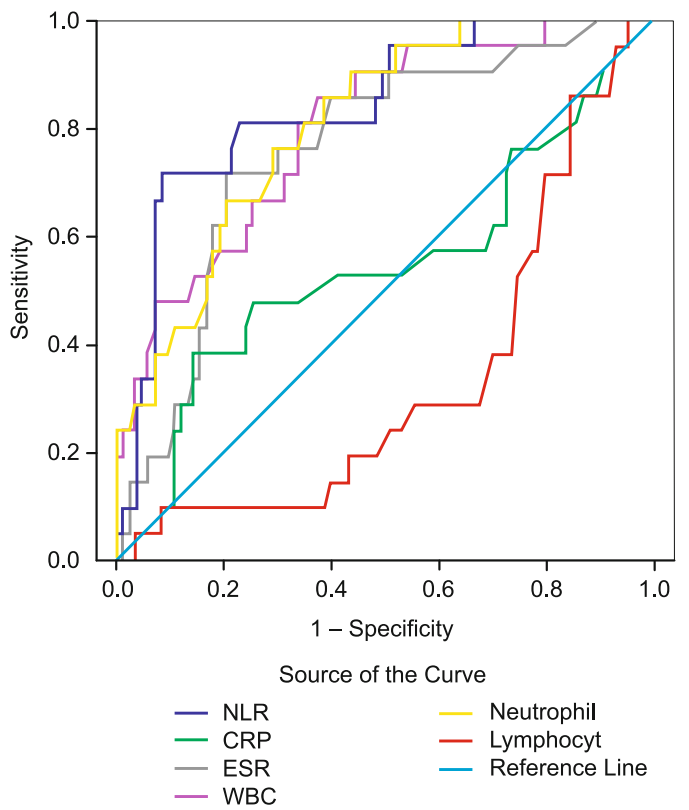

Fig. 1. Receiver operating characteristic (ROC) curve of neutrophil to lymphocyte ratio (NLR) vs other inflammation markers in predicting active disease for $\mathrm{CD}$.
Tab. 5. ROC analyses of NLR to differentiate active from inactive CD and UC.

\begin{tabular}{|c|c|c|c|c|c|c|c|}
\hline & & Cut-off & AUC & $\begin{array}{c}\text { Sensitivity } \\
(\%)\end{array}$ & $\begin{array}{c}\text { Specificity } \\
(\%)\end{array}$ & $\begin{array}{l}\text { NPV } \\
(\%)\end{array}$ & $\begin{array}{l}\text { PPV } \\
(\%)\end{array}$ \\
\hline $\mathrm{CD}$ & NLR & 3.2 & 83 & 81 & 59 & 74 & 93 \\
\hline$\overline{\mathrm{UC}}$ & NLR & 3.1 & 74 & 78 & 69 & 64 & 84 \\
\hline
\end{tabular}

AUC - area under curve, NPV - negative predictive value, $\mathrm{PPV}$ - positive predictive value, $\mathrm{CD}$ - Crohn disease, UC - ulcerative colitis, NS - non-significant.

summarized in Tables 1 and 2. The mean NLR values of active $\mathrm{CD}$ patients and controls were $5.25 \pm 1.85$ and $1.68 \pm 0.59$, respectively $(\mathrm{P}=0.000)$. The mean NLR values of inactive $\mathrm{CD}$ patients and controls were $1.78 \pm 0.32$ and $1.68 \pm 0.59 \mathrm{p}$ - inactive period) $\mathrm{CD}$ patients and controls are summarized in Table 1 . The mean NLR values of active UC patients and controls were $4.12 \pm 1.41$ and $1.68 \pm 0.59$, respectively $(\mathrm{p}=0.000)$. The mean NLR values of inactive UC patients and controls were $1.55 \pm 0.45$ and $1.68 \pm$ 0.59 , respectively, thus NS. The mean NLR and other inflammatory markers values of UC patients (active- inactive period) and controls are summarized in Table 2.

Table 3 shows that mean NLR values of active CD patients were significantly higher than those of inactive $\mathrm{CD}$ patients $(5.25$ \pm 1.85 vs $1.78 \pm 0.32, p=0.000)$. Mean NLR values of active UC patients were significantly higher than those of inactive UC patients ( $4.12 \pm 1.41$ vs $1.55 \pm 0.45, p=0.000)$. Inflammatory markers such as WBC, CRP and ESR, were found to be significantly elevated in active $\mathrm{CD}$ and $\mathrm{UC}$ compared to those in inactive $\mathrm{CD}$ and $\mathrm{UC}$.

Although Spearman correlation analysis in active $\mathrm{CD}$ indicated a significant correlation of NLR with WBC $(r=0.242, p=0.000)$ and neutrophil $(\mathrm{r}=0.882, \mathrm{p}=0.000)$, no correlation was found with CRP $(r=-0.61, p=0.793)$ and $\operatorname{ESR}(r=0.242, p=0.291)$.

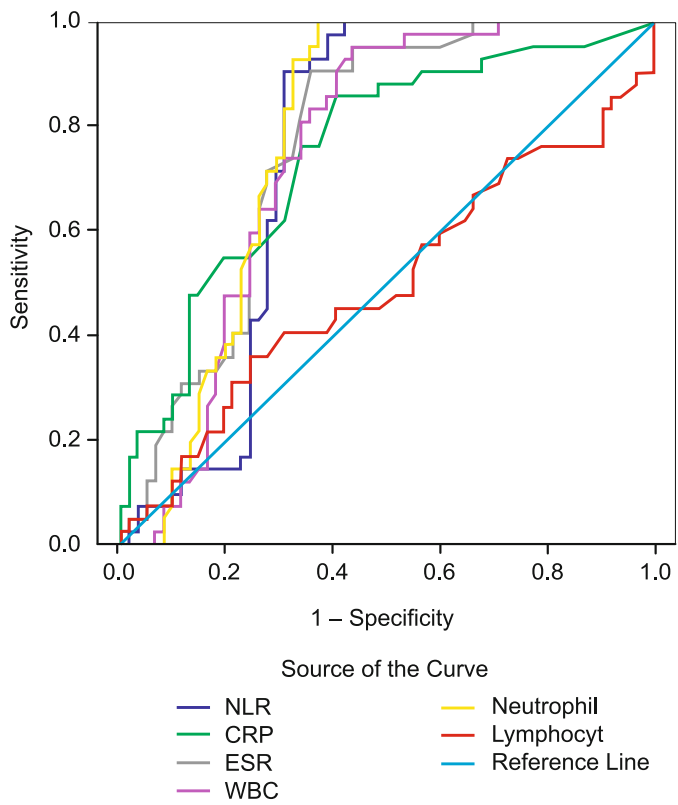

Fig. 2. Receiver operating characteristic (ROC) curve of neutrophil to lymphocyte ratio (NLR) vs other inflammation markers in predicting active disease for UC. 
Moreover, in inactive $\mathrm{CD}$, negative correlation was found with lymphocytes $(\mathrm{r}=-0.615, \mathrm{p}=0.007)$. While Spearman correlation analysis in active and inactive UC indicated a significant correlation of NLR with Neutrophil $(r=0.412, p=0.007),(r=0.515$, $\mathrm{p}=0.000)$, negative correlation was found with lymphocytes $(\mathrm{r}=$ $-0.615, \mathrm{p}=0.000),(\mathrm{r}=-0.451, \mathrm{p}=0.003)(\mathrm{Tab} .4)$.

ROC curve analysis suggested that the optimum NLR cut-off point for active CD was 3.2 with a sensitivity, specificity, PPV and NPV of $81 \%, 59 \%, 93 \%$, and $74 \%$, respectively (AUC - 0.830). ROC curve analysis suggested that the optimum NLR cut-off point for active UC was 3.1 with a sensitivity, specificity, PPV and NPV of $78 \%, 69 \%, 84 \%$, and $64 \%$, respectively (AUC - 0.740) (Tab. 5 , Figs 1 and 2).

\section{Discussion}

Our study's aim relates to the identification of NLR as a new noninvasive marker of disease severity in subjects with IBD. Our results demonstrate that values of NLR, CRP, ESR and WBC are higher in patients with active $\mathrm{CD}$ and UC compared with controls. The values of NLR, CRP and ESR were also found to be decreased after remission of CD and UC. Besides, the values of NLR and the other inflammatory markers were higher in patients with active $\mathrm{CD}$ and UC compared with those in remission period.

While the values of NLR were positively correlated with WBC and neutrophil in patients with active $\mathrm{CD}$, it had a negative correlation with lymphocyte in patients with inactive $\mathrm{CD}$. On the other hand, in patients with inactive or active UC it has been shown that while the values of NLR were positively correlated with neutrophil, they had a negative correlation with lymphocyte. Compared to inactive IBD and controls, high levels of N/L ratio in the sera of active IBD patients support the view that neutrophils may have a role in the inflammatory cascade of IBD and disease pathophysiology.

Although clinical, histological, radiologic and endoscopic examinations are used to determine disease activity in IBD patients, a large number of invasive and non-invasive methods have also been investigated for IBD diagnosis and determination of disease activity $(19,21)$. Although there is no ideal single serum marker for predicting disease severity, WBC, CRP and ESR are the most commonly used inflammatory indices in routine clinical practice for determining IBD activity. These parameters can change according to the degree of the inflammatory state, but they do not adequately reflect disease activity because of their low sensitivity and specificity for intestinal inflammation (22). Despite the better known role of inflammatory cells in IBD pathogenesis, there are a few data in literature describing the role of NLR as a predictor of disease severity. In this context, this study was designed to evaluate the role of NLR in prediction of IBD activation in conjunction with other clinical and biochemical severity indices. NLR was described in cardiac and non-cardiac disorders $(15,23)$. It was found that NLR was significantly higher in patients with chronic renal failure than in the control group $(24,25)$. Besides, it was found to be correlated with the activation of ulcerative colitis (26). In many diseases with chronic inflammation, NLR was found to be correlated with the levels of CRP, ESR and WBC, for example, in chronic renal failure and ulcerative colitis (23-26). NLR has been generally investigated as a prognostic index in inflammatory and neoplastic diseases such as colorectal cancer, pancreatic cancer, hepatocellular and metastatic renal cell carcinoma, (27-29). Furthermore, recent studies also suggest that NLR is useful for predicting survival following coronary interventions and non-ST-segment elevation myocardial infarction $(13,30,31)$. Moreover, based on the findings of our study, we believe that a standardized cut-off value for NLR in estimating disease severity is crucial for initial evaluation. Because of the need for starting an optimal treatment as soon as possible, elevated NLR levels can give a significant clue to the clinician for estimating disease severity.

The study has some limitations including crosssectional design and a relatively small sample size. Our study was not designed to elucidate the mechanistic pathways that lead to higher $\mathrm{N} / \mathrm{L}$ ratio in patients with IBD.

In brief, this study demonstrates that in subjects with IBD, the $\mathrm{N} / \mathrm{L}$ ratio is strongly associated with active disease and correlated with clinical and laboratory indices. The N/L ratio is an inexpensive and easily applicable test. Although the certainty of $\mathrm{N} / \mathrm{L}$ ratio for detecting active IBD is suboptimal, it can be measured easily and its combination with other markers assist to identify patients with increased risk of active disease. If our study can be taken forward with further trials, we think that a standardized cut-off value for NLR would simplify the diagnosis of IBD activation.

\section{References}

1. Xavier RJ, Podolsky DK. Unravelling the pathogenesis of inflammatory bowel disease. Nature 2007; 448: 427-434.

2. Abraham C, Cho JH. Inflammatory bowel disease. N Engl J Med 2009; 361: 2066-2078.

3. Jensen MD, Kjeldsen J, Nathan T. Fecal calprotectin is equally sensitive in Crohn's-disease affecting the small bowel and colon. Scand J Gastroenterol 2011; 46: 694-700.

4. Kane SV, Sandborn WJ, Rufo PA, Zholudev A, Boone J, Lyerly D, Camilleri M, Hanauer SB. Fecal lactoferrin is a sensitive and specific marker in identifying intestinal inflammation. Am J Gastroenterol 2003; 98: 1309-1314.

5. Kayazawa M, Saitoh O, Kojima K, Nakagawa K, Tanaka S, Tabata K, Matsuse R, Uchida K, Hoshimoto M, Hirata I, Katsuk. Lactoferrin in whole gut lavage fluid as a marker for disease activity in inflammatory bowel disease - comparison with other neutrophil-derived proteins. Am J Gastroenterol 2002; 97: 360-369.

6. Lewis JD. The utility of biomarkers in the diagnosis and therapy of inflammatory bowel disease. Gastroenterology 2011; 140: 1817-1826.

7. Naber AH, de Jong DJ. Assessment of disease activity in inflammatory bowel disease, relevance for clinical trials. Neth J Med 2003; 61: 105-110.

8. Peyrin-Biroulet L, Deltenre P, Suray N, Branche J, Sandborn WJ, Colombel JF. Efficacy and safety of tumor necrosis factor antagonists in Crohn's disease - meta-analysis of placebo-controlled trials. Clin Gastroenterol Hepatol 2008; 6: 644-653.

9. Sandborn WJ, Feagan BG, Hanauer SB, Lochs H, Löfberg R, Modigliani R, Present DH, Rutgeerts P, Schölmerik J, Stange EF, Suther- 
land LR. A review of activity indices and efficacy endpoints for clinical trials of medical therapy in adults with Crohn's disease. Gastroenterol 2002; 122: 512-530.

10. Caprilli R, Viscido A, Latella G. Current management of severe ulcerative colitis. Nat Clin Pract Gastroenterol Hepatol 2007; 4: 92-101.

11. Sandborn WJ, Loftus Jr EV, Colombel JF et al. Evaluation of serologic disease markers in a population-based cohort of patientswith ulcerative colitis and Crohn's disease. Inflamm Bowel Dis 2001; 7: 192-201.

12. Vermeire S, Van Assche G, Rutgeerts P. Laboratory markers in IBD - Useful, magic, or unnecessary toys? Gut 2006; 55: 426-431.

13. Duffy BK, Gurm HS, Rajagopal V, Gupta R, Ellis SG, Bhatt DL. Usefulness of an elevated neutrophil to lymphocyte ratio in predicting long-term mortality after percutaneous coronary intervention. Am J Cardiol 2006; 97: 993-996.

14. Rudiger A, Burckhardt OA, Harpes $P$ et al. The relative lymphocyte count on hospital admission is a risk factor for longterm mortality in patients with acute heart failure. Am J Emerg Med 2006; 24: 451-454.

15. Tamhane UU, Aneja S, Montgomery D et al. Association between admission neutrophil to lymphocyte ratio and outcomes in patients with acute coronary syndrome. Am J Cardiol 2008; 102: 653-657.

16. Nunez J, Nunez E, Bodi V et al. Usefulness of the neutrophil to lymphocyte ratio in predicting long-term mortality in ST segment elevation myocardial infarction. Am J Cardiol 2008; 101: 747-752.

17. Walsh SR, Cook EJ, Goulder F et al. Neutrophil-lymphocyte ratio as a prognostic factor in colorectal cancer. J Surg Oncol 2005; 91: 181-184.

18. Friedman GD, Tekawa I, Grimm RH et al. The leucocyte count correlates and relationship to coronary risk factors - the CARDIA study. Int J Epidemiol 1990; 19: 889-893.

19. Beyazit Y, Koklu S, Tas A, Purnak T, Sayilir A, Kurt M et al. Serum adenosine deaminase activity as a predictor of disease severity in ulcerative colitis. J Crohns Colitis 2012; 6: 102-107.

20. Travis SP. Review article - the management of mild to severe acute ulcerative colitis. Aliment Pharmacol Ther 2004; 20 (Suppl 4): 88r92.
21. Bruining DH, Loftus EV. Current and future diagnostic approaches - from serologies toimaging. Curr Gastroenterol Rep 2007; 9: 489-496.

22. Khan K, Schwarzenberg SJ, Sharp H, Greenwood D, WeisdorfSchindele S. Role of serology and routine laboratory tests in childhood inflammatory bowel disease. Inflamm Bowel Dis 2002; 8: 325-329.

23. Zahorec, R. Ratio of neutrophil to lymphocyte counts - rapid and simple parameter of systemic inflammation and stress in critically ill. Bratisl Lek Listy 2001; 1: 5-14.

24. Turkmen K, Guney I, Yerlikaya FH et al. The relationship between neutrophil-to-lymphocyte ratio and inflammation in end-stage renal disease patients. Ren Fail 2012; 34: 155-159.

25. Okyay GU, Inal S, Oneç K et al. Neutrophil to lymphocyte ratio in evaluation of inflammation in patients with chronic kidney disease. Ren Fail 2013; 35: 29-36.

26. Celikbilek M, Dogan S, Ozbakir O et al. Neutrophil-lymphocyte ratio as a predictor of disease severity in ulcerative colitis. J Clin Lab Anal 2013; 27 (1): 72-76.

27. Halazun KJ, Aldoori A, Malik HZ, Al-Mukhtar A, Prasad KR, Toogood GJ et al. Elevated preoperative neutrophil to lymphocyte ratio predicts survival following hepatic resection for colorectal liver metastases. Eur J Surg Oncol 2008; 34: 55-60.

28. Gomez D, Morris-Stiff G, Toogood GJ, Lodge JP, Prasad KR. Impact of systemic inflammation on outcome following resection for intrahepatic cholangiocarcinoma. J Surg Oncol 2008; 97: 513-518.

29. An X, Ding PR, Li YH, Wang FH, Shi YX, Wang ZQ et al. Elevated neutrophil to lymphocyte ratio predicts survival in advanced pancreatic cancer. Biomarkers 2010; 15: 516-522.

30. Azab B, Zaher M, Weiserbs KF, Torbey E, Lacossiere K, Gaddam $\mathbf{S}$ et al. Usefulness of neutrophil to lymphocyte ratio in predicting short and long term mortality after non-ST-elevation myocardial infarction. Am J Cardiol 2010; 106: 470-476.

31. Zahorec R. Ratio of neutrophil to lymphocyte count - rapid and simple parameter of systemic inflammation and stress in critically ill. Bratisl Med J 2001; 102 (1): 5 - 14. 